\title{
Pinch-off syndrome: a simple test is the best
}

\author{
Timothy D. Clay
}

Received: 15 November 2011 / Accepted: 22 January 2012/Published online: 5 February 2012

(C) SIMI 2012

\section{Introduction}

A 46-year-old woman presented to the emergency department (ED) with new onset atrial fibrillation (AF) with a rapid ventricular response. She had recently been diagnosed with carcinoma of the breast and had undergone breast conserving surgery. The pathological specimen showed a $25 \mathrm{~mm}$ moderately differentiated invasive ductal carcinoma. The sentinel node showed no evidence of metastatic spread. Pathologically the tumour was at stage IIA (T2 N0 M0). Estrogen and progesterone receptors were moderately positive by immunohistochemistry, and the human epidermal growth factor receptor 2 (HER-2) was positive by chromogenic in situ hybridisation (CISH). The patient had a prior history of developmental dysplasia of the hip. She had no personal or family history of cardiac disorders or cancer, was a lifelong non-smoker and consumed alcohol in moderation. Systemic chemotherapy with docetaxel, cyclophosphamide and trastuzumab was recommended to be followed by radiotherapy and hormonal therapy.

As venous access was difficult at the time of the first cycle of chemotherapy, a totally implanted venous device (TIVD, "Portacath") was inserted surgically on the day prior to the 2 nd cycle of chemotherapy. The line was inserted through a subclavian vein puncture using a landmark technique and intraoperative fluoroscopy, and the port was secured in a pocket under the skin. The postoperative chest X-ray study (CXR) demonstrated the position

T. D. Clay $(\bowtie)$

Department of Medical Oncology, St Vincent's Hospital, Cancer Centre, 1st Floor, Daly Wing, 35 Victoria Parade, Fitzroy, Melbourne, Victoria 3065, Australia

e-mail: timothy.clay@svhm.org.au of this device (Fig 1). The Portacath remained accessable after the procedure and was used for the 2nd cycle of chemotherapy.

On day 15 of the 2 nd cycle, the patient made contact with our oncology service complaining of dyspnoea and palpitations. An electrocardiogram (ECG) demonstrated new onset AF with a ventricular response rate of 100 beats per min. By the time of presentation to the ED, the patient had reverted to sinus rhythm. Cardiac enzymes levels were not elevated. The patient was not neutropenic, there were neither signs nor symptoms to suggest infection, and the thyroid function was normal. No evidence of pulmonary embolism (PE) was found on a CT pulmonary angiogram (CTPA). The patient was started on aspirin and atenolol. Echocardiography performed prior to discharge showed a structurally normal heart with an estimated ejection fraction of $60-65 \%$.

At the next visit to the chemotherapy suite, attempts were made to access the Portacath, but it was not possible to aspirate venous blood. A CXR was performed demonstrating fracture of the Portacath line, and migration of a segment to the right atrium (Fig 2), explaining the onset of the AF. Chemotherapy proceeded via peripheral access, and arrangements were made for the port to be removed. Under angiographic control, the right femoral vein was entered and attempts were made to snare the catheter fragment. The catheter fragment was in the atrioventricular groove, closely opposed to the tricuspid valve. Due to its position, attempts to remove it via the femoral approach were unsuccessful, and the procedure was halted. The patient returned 3 weeks later, and the catheter fragment was successfully removed via a right transjugular approach. A different angle of attack upon the catheter fragment allowed its removal. The remaining port and proximal catheter were removed surgically. Both parts of the port were submitted for analysis by 


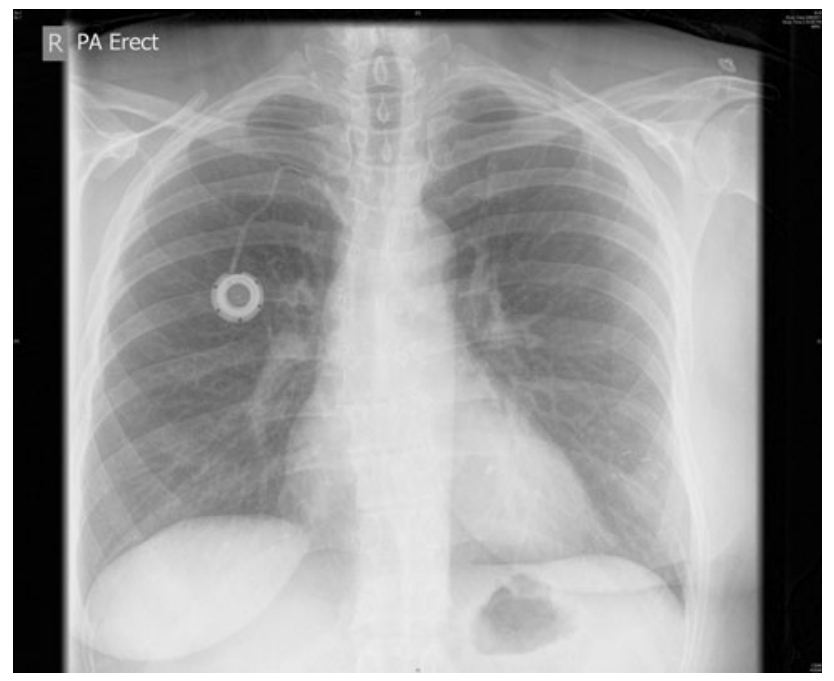

Fig. 1 Position of the "Portacath" on a chest X-ray study immediately after insertion

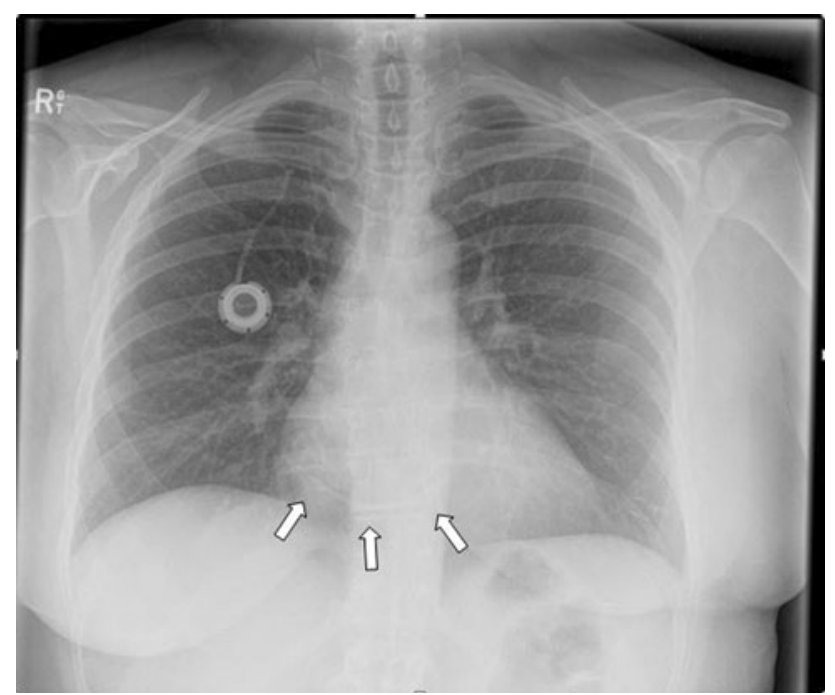

Fig. 2 A chest X-ray study shows the fracture of the "Portacath" catheter as it passes under the clavicle. The distal fragment is seen in the right atrium (arrows)

the manufacturer after removal. The patient has subsequently completed cytotoxic chemotherapy without further incident and will complete a full 12-month program of adjuvant trastuzumab.

\section{Discussion}

Long-term intravenous access devices are used in medical care for indications such as the administration of drugs, blood products, fluid and parenteral nutrition. Given the difficulties with venous access found in a number of oncology patients, together with the specific requirements of some treatment schedules, they are likely to remain in common use for many years to come. Our patient suffered from "pinch-off syndrome" (POS), which is one of the most common causes of catheter embolization from TIVDs. It occurs when a catheter is compressed between the clavicle and the first rib. POS can potentially be identified before failure of the TIVD by abnormal positioning on CXR, or difficulty administering and withdrawing fluids through the TIVD, which on occasion can improve with repositioning of the patient's arm [1]. Surov et al. [2] find that POS is the cause for catheter embolization (for all types of central venous access) in $40.9 \%$ of cases reported in their detailed review of the literature. Hinke et al. [1] provide a grading system for POS ranging from 0 to 3 where $0=$ normal, $1=$ abrupt change in course with no luminal narrowing of the catheter, $2=$ abrupt change in course with luminal narrowing and $3=$ catheter fracture. By Hinke's grading system our patient had grade 2 POS on a postinsertion CXR (Fig 1) and grade 3 POS on repeat CXR (Fig 2). The availability of a number of devices to interventional radiologists and cardiologists, such as snares and loops, means that embolised catheter fragments can be retrieved percutaneously, thereby avoiding the need for open surgery in most cases.

The occurrence of POS can occur from as early as the time of insertion to months or years later. A compilation of cases described in the literature by Mirza et al. [3] shows that in the 112 cases described, the average time that POS occurred was 5.3 months (range-immediate to 60 months). The authors of the papers referenced below discuss strategies for reducing the occurrence of POS [1-3]. Where appropriate, the internal jugular vein is preferred to the subclavian vein for insertion of the line. If the subclavian vein is used, it is preferable to access the vein lateral to the midclavicular line to reduce the amount of compression on the line. Care should be taken when handling the line at the time of insertion and removal. When the venous access device is no longer required, it should be expeditiously removed. Intraoperative radiographs can be taken to assess the position of the line with the shoulder in various positions. If significant POS is seen on a CXR postoperatively, or should it develop at a later time, the venous access device should be removed prior to the line fragmenting or fracturing. Damaged venous access devices should be submitted for materials analysis to ensure that a manufacturing fault has not contributed to device failure.

The use of ultrasound in inserting venous catheters into the subclavian vein has been shown to have a higher success rate with fewer complications when compared to the landmark method in a critically ill population [4]. This and other studies have focused on short-term complications in central catheters designed for short-term use. To date, there 
are no reviews in the literature that describe POS occurring after ultrasound guided insertion, or studies looking at whether the use of ultrasound may reduce the risk of POS.

The American College of Cardiology/American Heart Association/European Society of Cardiology Practice Guidelines provides evidence-based guidelines for the investigation and management of $\mathrm{AF}$ [5]. The guidelines recommend a thorough history and examination, and minimum baseline investigations of an ECG, transthoracic echocardiogram, and tests of thyroid, renal and hepatic function. Further investigations can be undertaken as required. Given a history of malignancy, recent surgery and chemotherapy, a PE was appropriately considered in the differential diagnosis for new onset AF. CTPA requires the injection of radio-opaque contrast material through a large peripheral vein using an automatic injector. In the case of our patient, the prior left sided breast cancer surgery meant that contrast needed to be injected through the right antecubital fossa. As the portacath and its line were radioopaque, like the intravenous contrast, the fracture of the portacath at the entry point into the subclavian vein and the right atrial fragment were not detected. Similarly, an echocardiogram did not identify the embolised catheter fragment. These tests were reviewed after the diagnosis of POS was made. The imaging changes were subtle and easily missed in the absence of clinical direction from the requesting clinician. There was potential for harm due to the delay in diagnosis.
It is important to follow a logical sequence in the investigation of patients with new symptoms. As demonstrated above, it is possible for complex expensive diagnostic tests to miss a simple diagnosis. A CXR should be undertaken in patients with TIVDs, and a new arrhythmia as it is a simple, rapid and cheap test that may identify device failure quickly. Had our patient had a CXR as her first investigation, it would have avoided the need for CTPA and echocardiogram, and allowed the issue to be addressed in a more timely fashion.

Conflict of interest None.

\section{References}

1. Hinke DH, Zandt-Stastny DA, Goodman LR et al (1990) Pinch-off syndrome: a complication of implantable subclavian venous access devices. Radiology 177(2):353-356

2. Surov A, Wienke A, Carter J et al (2009) Intravascular embolisation of venous catheter-causes, clinical signs and management. JPEN J Parenter Enteral Nutr 33(6):677-685

3. Mirza B, Vanek VW, Kupensky DT (2004) Pinch-off syndrome: case report and collective review of the literature. Am Surg 70(7):635-638

4. Fragou M, Gravvanis A, Dimitriou V et al (2011) Real-time ultrasound-guided subclavian vein cannulation versus the landmark method in critical care patients: a prospective randomised study. Crit Care Med 39:1607-1612

5. Fuster V, Ryden LE, Cannom DS et al (2006) ACC/AHA/ESC 2006 guidelines for the management of patients with atrial fibrillation. Circulation 114:e257-e354 